\title{
Three-dimensional MIMO- OTA calibration to achieve the Gaussian angular power spectra in elevation
}

\author{
Kazuhiro Honda ${ }^{\text {a) }}$ and Kun Li \\ Graduate School of Engineering, Toyama University, \\ 3190 Gofuku, Toyama-shi, Toyama 930-8555, Japan \\ a)hondak@eng.u-toyama.ac.jp
}

\begin{abstract}
This paper presents a calibration method for measuring the channel capacity using a three-dimensional multiple-input multiple-output (MIMO) Over-The-Air (OTA) apparatus. The reference level is calibrated to establish the Gaussian angular power spectra in elevation in consideration of various power losses that exist in the fading emulator. The measured results of MIMO channel capacity agree well with the analytical outcomes calculated by Monte Carlo simulation, demonstrating that the proposed calibration method gives a high degree of accuracy for measuring the channel capacity using the three-dimensional MIMO-OTA apparatus.
\end{abstract}

Keywords: three-dimensional fading emulator, MIMO-OTA, calibration, channel capacity

Classification: Antennas and Propagation

\section{References}

[1] C. Chong, F. Watanabe, K. Kitao, T. Imai, and H. Inamura, "Evolution trends of wireless MIMO channel modeling towards IMT-advanced," IEICE Trans. Commun., vol. E92-B, no. 9, pp. 2773-2788, Sep. 2009. DOI:10.1587/ transcom.E92.B.2773

[2] T. Sakata, A. Yamamoto, K. Ogawa, H. Iwai, J. Takada, and K. Sakaguchi, "A spatial fading emulator for evaluation of MIMO antennas in a cluster environment," IEICE Trans. Commun., vol. E97-B, no. 10, pp. 2127-2135, Oct. 2014. DOI:10.1587/transcom.E97.B.2127

[3] K. Honda, T. Kabeya, K. Karitani, K. Li, K. Ogawa, Y. Koyanagi, H. Sato, and R. Miura, "A method of realizing Gaussian angular power spectra in elevation using a three-dimensional fading emulator," IEICE Technical Report, AP2015197, pp. 37-42, Feb. 2016.

[4] K. Li, K. Honda, and K. Ogawa, "Three-dimensional over-the-air assessment for vertically arranged MIMO array antennas," IEICE Trans. Commun., vol. E99-B, no. 1, pp. 167-176, Jan. 2016. DOI:10.1587/transcom.2015EBP3148

[5] K. Honda, K. Li, and K. Ogawa, "A calibration method for the OTA testing of active MIMO antennas," IEICE Technical Report, AP2014-60, pp. 67-72, July 2014. 


\section{Introduction}

To realize ultra-large-capacity mobile communications, cellular systems operated in a small cell are being developed [1]. In the small cell systems, incident waves around a mobile terminal come from the elevation direction due to the short distance between the base station and the mobile terminal. Thus, the evaluation of terminal antennas in consideration of the incident wave characteristics in elevation is necessary. In previous study [2], the channel capacity of a multipleinput multiple-output (MIMO) array antenna is evaluated using a two-dimensional fading emulator. However, the incident waves coming from the elevation direction cannot be simulated due to the absence of the scatterers arranged in elevation.

We have proposed a three-dimensional Over-The-Air (OTA) apparatus [3], where there are two major factors need to be taken into account for ensuring a high degree of accuracy of the measured channel capacity. The first issue is the radiation characteristics of the reference antenna for measuring the incident wave power. The second issue is that we must consider various power losses arising from an OTA apparatus to realize the desired propagation environment.

This paper presents a calibration method for measuring the channel capacity using a three-dimensional MIMO-OTA apparatus. Firstly, the structure of the developed fading emulator is described. Then, the reference level is calibrated to establish the Gaussian angular power spectra in elevation in consideration of various power losses of microwave components comprising the fading emulator.

\section{Three-dimensional MIMO-OTA apparatus}

Fig. 1(a) shows the structure of the three-dimensional MIMO-OTA apparatus based on the analytical investigation [4] whereas Fig. 1(b) indicates its photograph. The angular power distribution of the incident wave is set to a uniform in azimuth and Gaussian in elevation. The desired power distribution in elevation is defined as the following equation:

$$
P_{g}(\theta)=\frac{1}{\sqrt{2 \pi} \sigma_{s}} \exp \left[-\frac{\left\{\theta-\left(\frac{\pi}{2}-m_{s}\right)\right\}^{2}}{2 \sigma_{s}^{2}}\right] \quad(0 \leq \theta \leq \pi)
$$

where $m_{s}$ represents the average elevation angle and $\sigma_{s}$ signifies the standard deviation.

In Fig. 1(a), the output signal of the network analyzer is separated into three stages corresponding to each elevation angle by the power divider in the incident wave control unit. The amplitude of each distributed signal is controlled using the attenuators $\left(A T T_{1}, A T T_{2}, A T T_{3}\right)$ to obtain the desired angular spread $\sigma_{s}$, as described in Eq. (1) [3].

Then, the signal of each elevation direction is distributed to the vertical and horizontal polarization components by the power divider in the XPR (cross-polarization power ratio) control unit, where the desired $X P R$ is realized by setting the appropriate values of the attenuators $\left(A T T_{\theta i}, A T T_{\phi i}\right)$. 


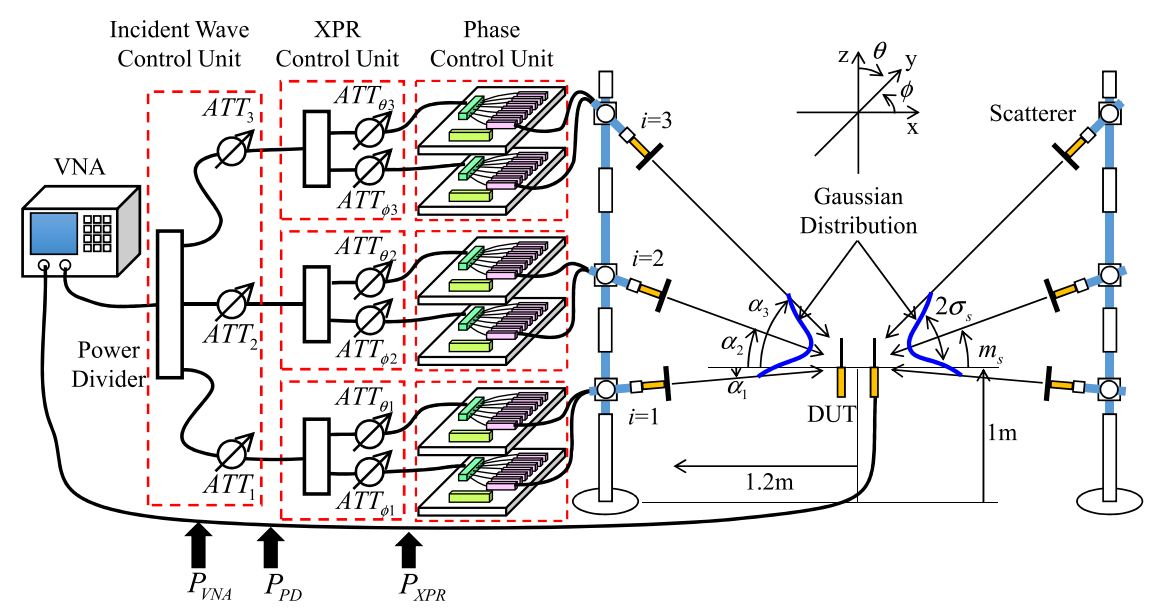

(a) Structure
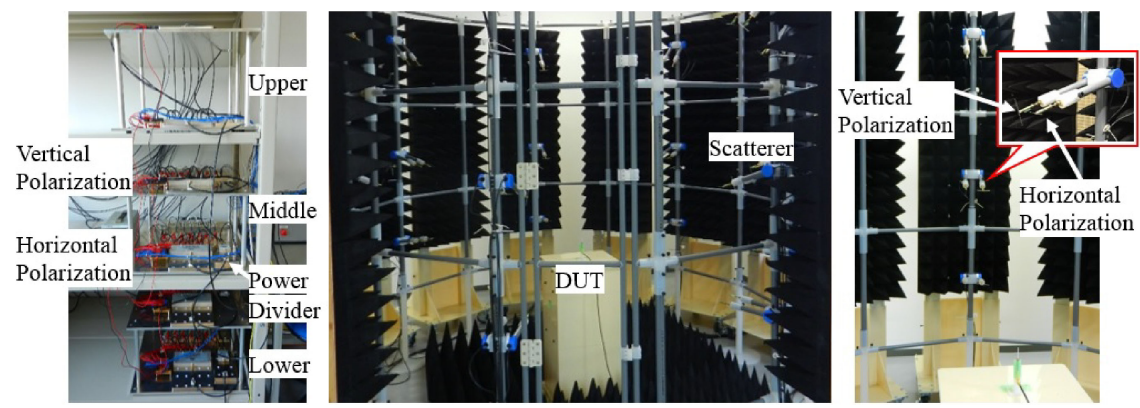

(b)Photograph

Fig. 1. Three-dimensional MIMO-OTA apparatus.

Finally, the signals of both polarization components are controlled by the phase shifters in the phase control unit. The radiated signals from the vertical and horizontal dipole antennas, illustrated in the right-hand picture of Fig. 1(b), are summed around the DUT antenna placed at the center of the emulator. Based on this apparatus, the transmission characteristics $\left(S_{21}\right)$ is measured by the network analyzer.

\section{Calibration method for MIMO channel capacity measurement}

When the MIMO channel capacity is measured, the signal to noise ratio $(S N R)$ is determined by

$$
S N R=\frac{R E F}{N}
$$

where $R E F$ is defined as the power received by an isotropic antenna for all radio waves from the scatterers [2]. $N$ is the power of the noise. However, since the isotropic antenna does not exist, the received power measured using other antennas must be compensated. Moreover, to realize the desired propagation environment, the power losses in the emulator must be considered.

We propose the calibration method of the reference level using a reference antenna as described by

$$
R E F=R E F_{d} G_{G} G_{X P R}
$$


where $R E F_{d}$ is the reference level considering the radiation gain of a reference antenna, $G_{G}$ and $G_{X P R}$ is the power loss in the incident wave and XPR control unit, respectively.

Firstly, we explain the reference level measurement. In the two-dimensional fading emulator, a half-wavelength dipole antenna is used for measuring the received level, and that value is calibrated based on the maximum radiation gain of dipole antenna in the horizontal plane, i.e., $2.15 \mathrm{~dB}$ [5]. In a similar way, the dipole antenna is used for measuring the received level of the three-dimensional fading emulator. However, different from the two-dimensional measurement in [5], the three-dimensional radiation characteristics of the dipole antenna must be taken into account.

The average received power $E\left[\left|S_{21}\right|^{2}\right]$ of the dipole antenna placed at the center of the emulator is measured using the network analyzer when only the scatterers with the vertical polarization component are used and $\sigma_{s}$ is set to infinity. Then, the reference level is calculated using $E\left[\left|S_{21}\right|^{2}\right]$ by

$$
R E F_{d}=\frac{E\left[\left|S_{21}\right|^{2}\right]}{G_{d}}
$$

where $G_{d}$ is the sum of the radiation gain of a half-wavelength dipole antenna, derived from the following procedures.

For the sake of simplicity, we considered the radiation gain in the direction of each incident wave coming from the scatterers located at the different elevation. Fig. 2(a) shows the three-dimensional picture of the radiation gain of dipole antenna calculated by the method of moments. The ratio of the received power of the dipole antenna and that of the isotropic antenna is determined by

$$
G_{d}=\frac{\sum_{i=1}^{3} P_{r i \_ \text {dipole }}}{\sum_{i=1}^{3} P_{\text {ri_isotropic }}}=\frac{\sum_{i=1}^{3} P_{g i} G_{r i \_d i p o l e}}{\sum_{i=1}^{3} P_{g i} G_{r i \_ \text {isotropic }}}
$$

where $P_{r i}$ is the received power, $P_{g i}$ is the incident wave power. $G_{r i}$ is the radiation gain toward the incident wave from the $i$-th scaterer angle in elevation. When the reference level is measured, the propagation environment is set to the uniform distribution in elevation to allow the incident wave power of each elevation direction to be equal. Hence, Eq. (5) is reduced to the following equation:

$$
G_{d}=\frac{\sum_{i=1}^{3} G_{r i \_ \text {dipole }}}{\sum_{i=1}^{3} G_{\text {ri_isotropic }}}=\frac{1}{3} \sum_{i=1}^{3} 1.64 \frac{\cos ^{2}\left\{\frac{\pi}{2} \cos \left(\frac{\pi}{2}-\alpha_{i}\right)\right\}}{\sin ^{2}\left(\frac{\pi}{2}-\alpha_{i}\right)}
$$

where $\alpha_{i}$ is the elevation angle of scatterers, as shown in Fig. 1(a) and Fig. 2(a).

Secondly, we consider the power losses for realizing the Gaussian angular power spectra in elevation, defined as Eq. (1). Fig. 2(b) shows the configuration of incident wave control unit. By adjusting the appropriate values of the attenuators $\left(A T T_{1}, A T T_{2}, A T T_{3}\right)$, the desired $\sigma_{s}$ in Eq. (1) is realized. To this end, the insertion loss of attenuators needs to be compensated as described in the following equation: 


$$
G_{G}=\frac{P_{P D}}{P_{V N A}}=\frac{\sum_{i=1}^{3} P_{P D i}}{P_{V N A}}
$$

where $P_{V N A}$ and $P_{P D}$ is the input and output power of the incident wave control unit, as shown in Fig. 2(b).

As mentioned above, when the reference level is measured, only the vertical polarization component is considered. However, the polarization characteristics, denoted by $X P R$, must be established in the emulator using the XPR control unit shown in Fig. 2(c) when a mobile terminal is measured in a realistic cellular propagation environment.

In Fig. 2(c), the XPR control unit comprises power divider, attenuators, and coaxial cables. Hence, the reference level must be calibrated considering the power losses in the XPR control unit, as expressed by

$$
G_{X P R}=\frac{P_{X P R}}{P_{P D}}=\frac{\sum_{i=1}^{3} P_{X P R i}}{\sum_{i=1}^{3} P_{P D i}}
$$

where $P_{P D}$ and $P_{X P R}$ is the input and output power of the XPR control unit, respectively. The total output power of both polarization components is calculated as

$$
P_{X P R i}=P_{\theta i}+P_{\phi i}
$$

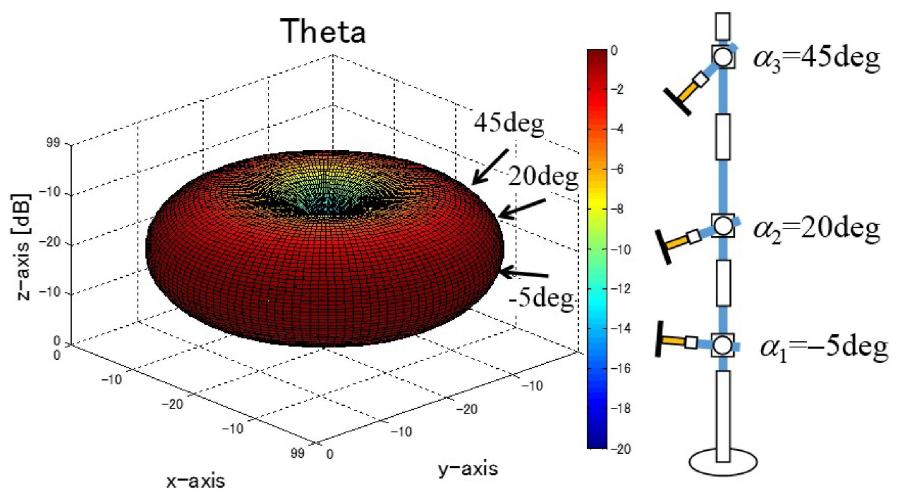

(a) Gain difference of the reference dipole antenna in elevation

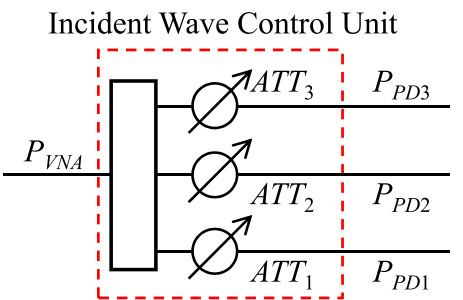

(b) Power loss in the

incident wave control unit

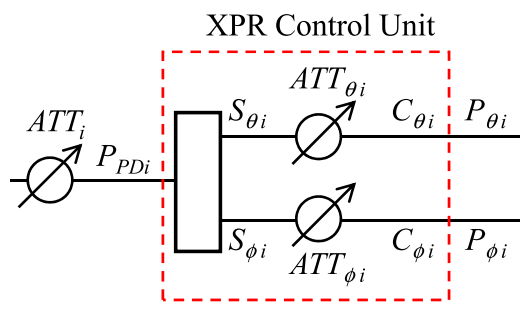

(c)Power loss in the XPR control unit

Fig. 2. Key factors that must be considered when the calibration of a three-dimensional MIMO-OTA is performed. 
where $P_{\theta i}$ and $P_{\phi i}$ are the output power of vertical and horizontal polarization components, respectively. $P_{\theta i}$ and $P_{\phi i}$ are calculated considering the power loss of each component by

$$
\begin{aligned}
P_{\theta i} & =P_{P D i} S_{\theta i} A T T_{\theta i} C_{\theta i} \\
P_{\phi i} & =P_{P D i} S_{\phi i} A T T_{\phi i} C_{\phi i}
\end{aligned}
$$

where $S_{\theta i}$ and $S_{\phi i}$ are the ratio of the input and output power of the power divider in the vertical and horizontal polarization components, respectively. $A T T_{\theta i}$ and $A T T_{\phi i}$ are the insertion loss of attenuator in each polarization component. $C_{\theta i}$ and $C_{\phi i}$ are the power loss of coaxial cable in each polarization component.

\section{Experimental results}

To confirm the validity of the proposed calibration method, channel capacity measurements have been carried out using the developed MIMO-OTA apparatus [3]. The measurement was conducted using a 4-element half-wavelength dipole array antenna placed at the center of the emulator, as shown in Fig. 3(a). The distance between the array elements was $9 \mathrm{~cm}$. The frequency was set to $2 \mathrm{GHz}$. $S N R$ was set to $30 \mathrm{~dB}$.

Firstly, the proposed calibration method is verified when $\sigma_{s}$ is varied. Fig. 3(b) shows the measured and analytical results of $4 \times 4 \mathrm{MIMO}$ channel capacity with $\sigma_{s}$ changing from 10 degrees to infinity. $X P R$ is set to $10 \mathrm{~dB}$. In Fig. 3(b), the symbols - show the measured results obtained from the emulator while the curve indicates the analytical outcome calculated by the Monte Carlo simulation [4]. As can be seen in Fig. 3(b), the measured results agree well with the analytical outcomes. Therefore, the evaluation of channel capacity considering the Gaussian incident wave in elevation can be realized using the proposed calibration method. When
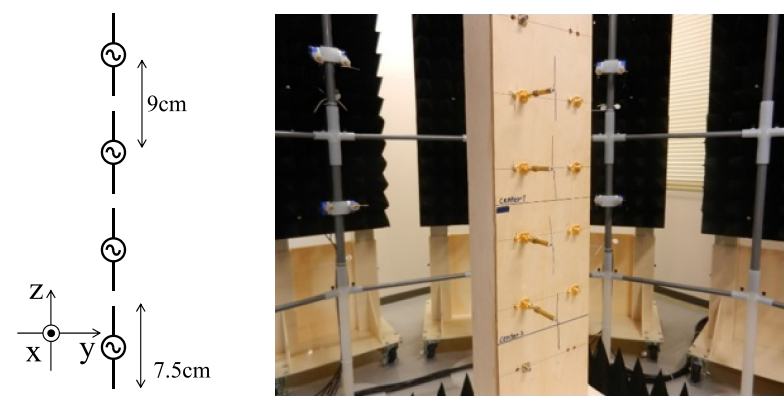

(a) 4-element vertically arranged MIMO array antenna

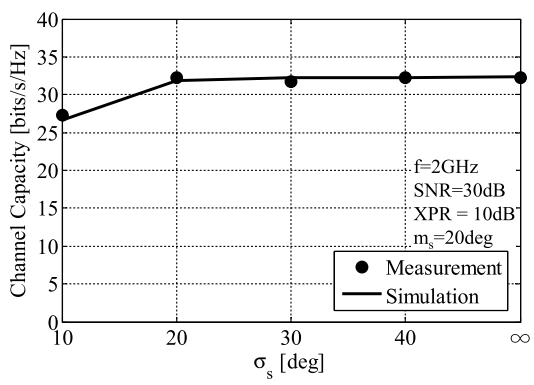

(b) MIMO channel capacity vs. $\sigma_{s}$

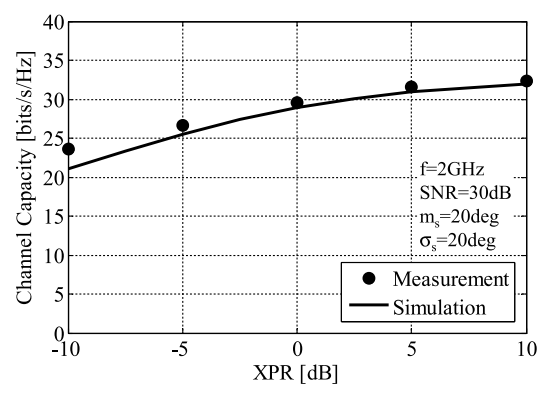

(c) MIMO channel capacity vs. XPR

Fig. 3. Experimental verification of the proposed calibration method. 
$\sigma_{s}=10$ degrees, the channel capacity is degraded because the narrow angular spread results in a high correlation [4].

Then, the proposed calibration method is evaluated when $X P R$ is varied. Fig. 3(c) shows the channel capacity with $X P R$ varing from -10 to $10 \mathrm{~dB} . \sigma_{s}$ is set to $20 \mathrm{deg}$. In Fig. 3(c), the channel capacity is varied depending on $X P R$, indicating that calibration method considering the power loss in the XPR control unit is effective.

\section{Conclusion}

This paper presents a calibration method for measuring the MIMO channel capacity using the three-dimensional fading emulator. The measured results have a high degree of accuracy compared with the analytical outcomes, demonstrating that the calibration method for measuring the channel capacity using the MIMO-OTA apparatus is valid.

\section{Acknowledgments}

This work was entrusted by the MIC-SCOPE 2015. 